\title{
Universal decay of scalar turbulence
}

\author{
M. Chaves ${ }^{1}$, G. Eyink ${ }^{2}$, U. Frisch ${ }^{1}$ and M. Vergassola ${ }^{1}$ \\ ${ }^{1}$ CNRS, Observatoire de la Côte d'Azur, B.P. 4229, 06304 Nice Cedex 4, France. \\ 2 Department of Mathematics, Univ. of Arizona, Tucson, AZ85721, USA.
}

(November 9, 2018)

\begin{abstract}
The asymptotic decay of passive scalar fields is solved analytically for the Kraichnan model, where the velocity has a short correlation time. At long times, two universality classes are found, both characterized by a distribution of the scalar - generally non-Gaussian - with global self-similar evolution in time. Analogous behavior is found numerically with a more realistic flow resulting from an inverse energy cascade.
\end{abstract}

PACS number(s) : 47.10.+g, 47.27.-i, 05.40.+j

Recent research on passive scalars in prescribed turbulent flows has mostly concentrated on universal smallscale properties in the presence of large-scale random pumping of the scalar (see Ref. [i] and references therein). Another important problem is the decay of a scalar field $\theta(\boldsymbol{r}, t)$ with prescribed random initial conditions. It is then possible to obtain a stronger form of universality at all scales, but only at long times, when (most) of the information in the initial conditions has been washed out. Specifically, we shall be interested in self-similar decay at infinite (or very large) Péclet numbers. We ask the following questions: (i) Is it possible to find time-dependent rescaling factors of length and scalar amplitude such that in the rescaled variables the full single-time probability of the passive field becomes independent of time as $t \rightarrow \infty$ ? (ii) If the first question holds true, what are the universality classes (with respect to the initial conditions) of the asymptotically self-similar scalar field and what are their statistical properties? Such issues are of course not limited to passive scalars. For Burgers turbulence, a systematic theory of self-similar decay giving, e.g., the law of decay of the energy can be derived [2 伍. We shall return to the corresponding issues for 3-D Navier-Stokes turbulence in the conclusions. Here we shall first address such issues within the framework of the Kraichnan model [5] of passive scalars in which the velocity has a very short correlation time. This allows analytical and numerical work shedding light on the basic mechanisms of scalar decay. We show that there are two universality classes, both generally having self-similar non-Gaussian distributions. Spatially smooth and very rough velocity fields represent two limiting special cases: for the former self-similar decay is broken [6,7] while for the latter nearly Gaussian self-similar decay obtains. We then turn, using numerical simulations, to decay in the presence of more realistic velocity fields for a scalar transported by a $2-\mathrm{D}$ velocity field arising from an inverse energy cascade process [8]. This also leads to self-similar non-Gaussian decay with exponential tails in the single-point scalar pdf.

The advection-diffusion equation governing the evolution of a passive scalar field is

$$
\partial_{t} \theta(\boldsymbol{r}, t)+\boldsymbol{v}(\boldsymbol{r}, t) \cdot \nabla \theta(\boldsymbol{r}, t)=\kappa \nabla^{2} \theta(\boldsymbol{r}, t),
$$

where $\boldsymbol{v}(\boldsymbol{r}, t)$ is the incompressible advecting flow and $\kappa$ is the molecular diffusivity. Eq. (11) governs the evolution of the probability density at position $r$ and time $t$ of tracer particles. Their Lagrangian trajectories $\boldsymbol{\rho}(t)$ obey the stochastic equation

$$
d \boldsymbol{\rho}(t)=\boldsymbol{v}(\boldsymbol{\rho}(t), t) d t+\sqrt{2 \kappa} d \boldsymbol{\beta}(t),
$$

where $\boldsymbol{\beta}(t)$ is the $d$-dimensional isotropic Brownian motion. The scalar correlation functions at time $t$ can then be expressed in terms of the initial ones as

$$
\begin{aligned}
C_{N}(\underline{\boldsymbol{r}}, t) & =\left\langle\theta\left(\boldsymbol{r}_{1}, t\right) \ldots \theta\left(\boldsymbol{r}_{N}, t\right)\right\rangle \\
& =\int \mathcal{P}_{N}(\underline{\boldsymbol{\rho}}, 0 \mid \underline{\boldsymbol{r}}, t) C_{N}(\underline{\boldsymbol{\rho}}, 0) d \underline{\boldsymbol{\rho}} .
\end{aligned}
$$

Here, $\underline{\boldsymbol{r}}$ denotes the set $\boldsymbol{r}_{1}, \ldots, \boldsymbol{r}_{N}$ and $\mathcal{P}_{N}$ is the probability density that $N$ Lagrangian particles, being at $\underline{r}$ at time $t$, were previously at $\boldsymbol{\rho}$ at time $t=0$. The initial condition $\theta(\boldsymbol{r}, 0)$ is taken random and statistically homogeneous with correlation length $L$. The statistical homogeneity implies that the absolute position of the particles is irrelevant and only the separation variables $\boldsymbol{r}_{i, j}=\boldsymbol{r}_{i}-\boldsymbol{r}_{j}$ matter. In the sequel we shall always consider the propagator in the restricted homogeneous sector, i.e. in the $(N-1) d$ separation variables. Note that the evolution equation (11) conserves the so-called Corrsin integral [9]

$$
J_{0}=\int C_{2}(\boldsymbol{r}, t) d \boldsymbol{r} .
$$

That conservation law alone does not however allow to predict the scalar energy decay: for this, we also need to know the growth of the scalar correlation length with time. This is where the specific properties of the advecting velocity come into play.

Let us begin with the Kraichnan model: the velocity is statistically homogeneous and stationary, isotropic and Gaussian. The second-order correlation function of its increments is

$$
\begin{array}{r}
D_{\alpha \beta}(\boldsymbol{r}, t) \equiv\left\langle v_{\alpha}(\boldsymbol{r}, t) v_{\beta}(\boldsymbol{r}, 0)\right\rangle-\left\langle v_{\alpha}(\boldsymbol{r}, t) v_{\beta}(\mathbf{0}, 0)\right\rangle= \\
D r^{-\gamma}\left[r^{2}(\gamma+d+1) \delta_{\alpha \beta}-(2-\gamma) r_{\alpha} r_{\beta}\right] \delta(t),
\end{array}
$$


where $\gamma$ controls the roughness of the field. Smooth flows correspond to $\gamma=0$, while for $\gamma=2$ the opposite limit of very rough fields is achieved. Hereafter, we shall concentrate on the generic non-smooth case $\gamma \neq 0$. The growth of the scalar length-scale is determined dimensionally to be $L(t) \sim t^{1 / \gamma}$ and the units are chosen for convenience to set $L=D=1$. Note that the velocity field in the Kraichnan model is statistically invariant under time inversion, i.e. $\boldsymbol{v}(\boldsymbol{r}, t)$ has the same statistical properties as $-\boldsymbol{v}(\boldsymbol{r},-t)$. It follows that the $N$-particle propagator satisfies

$$
\mathcal{P}_{N}(\underline{\boldsymbol{\rho}}, 0 \mid \underline{\boldsymbol{r}}, t)=\mathcal{P}_{N}(\underline{\boldsymbol{\rho}}, t \mid \underline{\boldsymbol{r}}, 0)=\mathcal{P}_{N}(\underline{\boldsymbol{r}}, t \mid \underline{\boldsymbol{\rho}}, 0) .
$$

The major consequence of the short correlation time assumption is that the Lagrangian trajectories are a Markov stochastic process and that the $N$-particle propagators $\mathcal{P}_{N}$ obey Fokker-Planck equations (see, e.g., Ref. [1]):

$$
\partial_{t} \mathcal{P}_{N}+\sum_{i \neq j} \nabla_{\alpha}^{r_{i}} \nabla_{\beta}^{r_{j}}\left[\kappa \delta_{\alpha \beta}+\frac{1}{2} D_{\alpha \beta}\left(\boldsymbol{r}_{i, j}\right)\right] \mathcal{P}_{N}=0 .
$$

The advective term in (11) conserves all the moments $\left\langle\theta^{n}\right\rangle$. The scalar decay is therefore due to the effects of the molecular dissipation and, as usual in turbulence, we are interested in the singular limit $\kappa \rightarrow 0$ (infinite Péclet numbers). The scalar dissipation $\kappa\left\langle(\nabla \theta)^{2}\right\rangle$ remains indeed finite in the limit. From the Lagrangian point of view, the molecular noise in (2) can be neglected and the singularity of the limit shows up in the fact that initially coinciding particles separate in finite time 110,11]. For $\kappa \rightarrow 0$, the fundamental solution of (7) is self-similar and the propagator possesses the scaling property

$$
\mathcal{P}_{N}\left(\lambda \underline{\boldsymbol{\rho}}, 0 \mid \lambda \underline{\boldsymbol{r}}, \lambda^{\gamma} t\right)=\lambda^{-(N-1) d} \mathcal{P}_{N}(\underline{\boldsymbol{\rho}}, 0 \mid \underline{\boldsymbol{r}}, t) .
$$

The simplest objects to investigate are the single-point moments $\left\langle\theta^{2 n}\right\rangle(t)$ and we are interested in their long-time behavior $t \gg 1$. The first universality class is formed by the initial conditions where the Corrsin invariant $J_{0}$ defined in (1) does not vanish. We shall also make the natural assumption that the initial scalar correlations decay rapidly enough to ensure integrability of the cumulants of arbitrary order. The long-time behavior of the singlepoint moments is obtained by setting $\boldsymbol{r}=0$ in (3) and using (8) to rescale out the time dependence. The resulting object involves $t^{d / \gamma} C_{2}\left(\boldsymbol{\rho} t^{1 / \gamma}, 0\right)$ which tends to $\delta(\boldsymbol{\rho}) J_{0}$. The final result is

$$
\begin{aligned}
\left\langle\theta^{2 n}\right\rangle(t) & \simeq \frac{J_{0}^{n}}{t^{n d / \gamma}}(2 n-1) ! ! \\
& \times \int \mathcal{P}_{2 n}\left(\boldsymbol{\rho}_{1}, \boldsymbol{\rho}_{1}, \ldots \boldsymbol{\rho}_{n}, \boldsymbol{\rho}_{n}, 1 \mid \underline{\mathbf{0}}, 0\right) d \boldsymbol{\rho}_{1} \ldots d \boldsymbol{\rho}_{n},
\end{aligned}
$$

as is easily seen for the special case of initial Gaussian statistics. In fact, it is not hard to show that the same

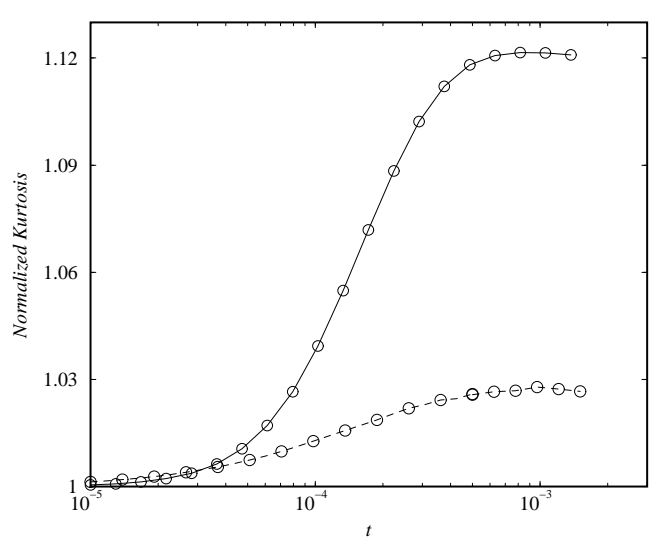

FIG. 1. The normalized kurtosis $\left\langle\theta^{4}\right\rangle / 3\left\langle\theta^{2}\right\rangle^{2}$ vs time in the Kraichnan model for the two values $\gamma=5 / 4$ (dashed line) and $\gamma=2 / 3$ (solid line). The error bars are comparable to the size of the symbols.

result holds for all initial data in the universality class $J_{0} \neq 0$ and rapid correlation decay. The integrability condition on the cumulant of order $2 n$ implies indeed that it must depend on all the $(2 n-1)$ independent interparticle separations. In the limit of long times, each dependence brings a function $\delta\left(\boldsymbol{\rho}_{i j}\right)$ and a factor $t^{d / \gamma}$ in the denominator. The total non-Gaussian contribution from the initial conditions will therefore decay faster than $t^{-n d / \gamma}$. Note that this argument implies a stronger form of universality than in forced steady states: the asymptotic pdf is here independent of the initial data, whereas in the steady states the scalar pdf depends on the details of the force. The expression (9) shows that the scalar statistics is self-similar in time. For example, the single point pdf $\mathcal{P}(\theta, t)$ takes the form $t^{d / 2 \gamma} Q\left(\theta t^{d / 2 \gamma}\right)$. The propagator in the limit of initially coinciding points that appears in (9) is a regular function of its arguments [10]. The smooth case $\gamma=0$ is special in this respect since nearby particles separate exponentially and their separation is proportional to its initial value. This is the physical reason why (9) and the ensuing self-similarity of the scalar distribution do not hold for $\gamma=0$ (see Refs. [6, /7). Note that the asymptotic pdf $\mathcal{P}(\theta, t)$, although universal and self-similar, is generally non-Gaussian. From (9), the hypothesis of Gaussianity is equivalent to the factorization of the probability for the $2 n$ particles to collapse into pairs, i.e. the integral in (9) should be equal to $\left[\int \mathcal{P}_{2}(\boldsymbol{\rho}, \boldsymbol{\rho}, 1 \mid \underline{\mathbf{0}}, 0) d \boldsymbol{\rho}\right]^{n}$. This is generally not the case, due to the correlations existing among the particle trajectories. The only exception is $\gamma=2$ where the particles are independent. The degree of non-Gaussianity is thus expected to decrease when $\gamma$ increases, as confirmed in Fig. 1. The simulations were performed by the Lagrangian method presented in Refs. 11, 12].

The second universality class is for initial conditions with Corrsin invariant $J_{0}=0$. From the realizability of 


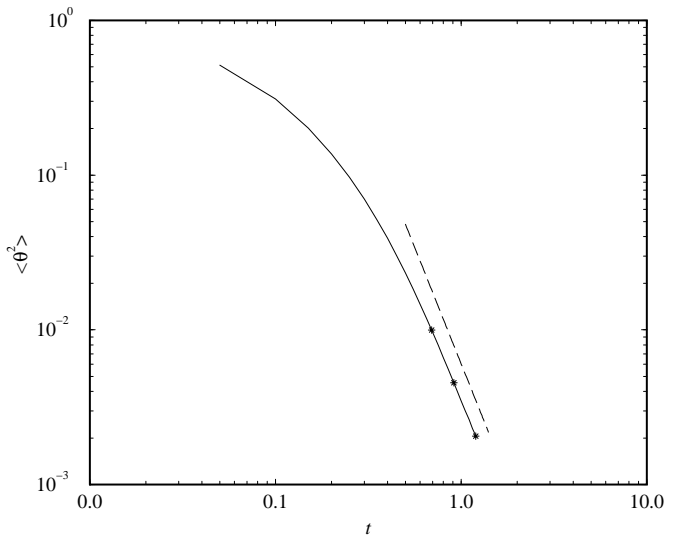

FIG. 2. The scalar energy $\left\langle\theta^{2}\right\rangle$ vs $t$ for the advection by a $2 \mathrm{D}$ flow generated by an inverse energy cascade. The dashed line is the asymptotic power law behavior $1 / t^{3}$.

the field it follows that the integral $J_{1}=\int r^{\gamma} C_{2}(\boldsymbol{r}) d \boldsymbol{r}$ is non-vanishing [13]. It is also easily checked using (3) and (7) that the integral remains invariant in time. $J_{0}=$ 0 implies that the integral of the correlation $C_{N}$ with respect to any of its arguments vanishes. Furthermore, the single-point moments can then be expressed as

$$
\left\langle\theta^{2 n}\right\rangle(t)=\int \mathcal{Q}_{2 n}(\underline{\boldsymbol{\rho}}, t \mid \underline{\boldsymbol{r}}, 0) C_{2 n}(\underline{\boldsymbol{\rho}}, 0) d \underline{\boldsymbol{\rho}} .
$$

Here

$$
\mathcal{Q}_{2 n}(\underline{\boldsymbol{\rho}}, t \mid \underline{\boldsymbol{r}}, 0) \equiv\left(\prod_{i=2}^{2 n} \Delta_{\boldsymbol{\rho}_{i, 1}, \mathbf{0}}\right) \mathcal{S}_{2 n} \mathcal{P}_{2 n}(\underline{\boldsymbol{\rho}}, t \mid \underline{\boldsymbol{r}}, 0),
$$

$\mathcal{S}_{2 n}$ denotes the symmetrizer in the $\boldsymbol{\rho}$ variables and the difference operator $\Delta$ acts on a function $f(\boldsymbol{x})$ as $\Delta_{\boldsymbol{x}}, \boldsymbol{y} f(\boldsymbol{x})=f(\boldsymbol{x})-f(\boldsymbol{y})$. To obtain (10), we set $\underline{\boldsymbol{r}}=0$ in (3), symmetrize in the $\boldsymbol{\rho}$ variables and use the condition $J_{0}=0$ to reexpress the result in terms of difference operators. For this, note that whenever any of the $\boldsymbol{\rho}$ variables in the propagator is replaced by $\mathbf{0}$ the integral vanishes. The long-time behavior of the single-point moments is then derived from (10) exploiting again the scaling property (8). For Gaussian initial conditions, we obtain

$$
\begin{aligned}
& \left\langle\theta^{2 n}\right\rangle(t) \simeq \frac{J_{1}^{n}}{t^{n(d / \gamma+1)}}(2 n-1) ! ! \times \\
& \lim \int \frac{\mathcal{Q}_{2 n}\left(\boldsymbol{\rho}_{1}, \boldsymbol{\rho}_{2}, \ldots \boldsymbol{\rho}_{2 n-1}, \boldsymbol{\rho}_{2 n}, 1 \mid \underline{\mathbf{0}}, 0\right)}{\left[\rho_{1,2} \ldots \rho_{2 n-1,2 n}\right]^{\gamma}} \prod_{i=1}^{n} d \boldsymbol{\rho}_{2 i-1},
\end{aligned}
$$

where we used the convergence of $\lambda^{d / \gamma}(\lambda \rho)^{\gamma} C_{2}(\lambda \boldsymbol{\rho}, 0)$ to $\delta(\boldsymbol{\rho}) J_{1}$ for large $\lambda$ 's. The limit of $\rho_{1,2} \ldots \rho_{2 n-1,2 n}$ tending to zero, which is understood in (12), has been shown to be well defined 14]. The strong universality of the scalar distribution is proved as for the $J_{0} \neq 0$ case. The expression (12) indicates that the asymptotic scalar distribution is again self-similar although the rescaling factor is now $t^{d /(\gamma+1)}$, in agreement with the exact solution for the second-order case [13].

The long-time behavior of multi-point scalar correlations can be derived from (3) as for the single-point moments. Using the scaling property (8) we are generally led to analyze $\mathcal{P}_{N}\left(\boldsymbol{\rho}, 1 \mid \underline{\boldsymbol{r}} / t^{1 / \gamma}, 0\right)$, i.e. the limit where the $N$ particles are initially close. This limit is crucial also for the small-scale scaling properties in the presence of pumping and leads to an asymptotic expansion for the propagator 10]

$$
\mathcal{P}_{N}(\underline{\boldsymbol{\rho}}, t \mid \lambda \underline{\boldsymbol{r}}, 0) \simeq \sum_{a, k} \lambda^{p_{a}+\gamma k} f_{a, k}(\underline{\boldsymbol{r}}) g_{a, k}(\underline{\boldsymbol{\rho}}, t),
$$

valid for small $\lambda$. The first sum is over the zero modes $f_{a} \equiv f_{a, 0}$ with scaling dimensions $p_{a}$ (in increasing order). The functions $f_{a, k}$ for different $k$ form the corresponding towers of the so-called slow modes. The leading term in the expansion (13) comes from the constant zero mode $f_{0,0}=1$ which corresponds to $g_{0,0}(\underline{\boldsymbol{\rho}}, t)=$ $\mathcal{P}_{N}(\underline{\boldsymbol{\rho}}, t \mid \underline{\mathbf{0}}, 0)$, i.e. the propagator for $N$ particles that are initially at the same position. Inserting (13) into (3) and using the dominance of $g_{0,0}$ is is easy to check that generic multi-point correlations will asymptotically decay as the corresponding single-point moment. Subdominant terms emerge by combining the correlation functions to cancel the dominant contribution. The classical example is given by the structure functions $S_{2 n}(\boldsymbol{r}, t)=\left\langle[\theta(\boldsymbol{r}, t)-\theta(\mathbf{0}, t)]^{2 n}\right\rangle$, related to the correlation functions by

$$
S_{2 n}(\boldsymbol{r}, t)=\left(\prod_{i=1}^{2 n} \Delta_{\boldsymbol{r}_{i}, \mathbf{0}}\right) C_{2 n}(\underline{\boldsymbol{r}}, t) .
$$

The difference operator $\Delta$ was defined in (11). The crucial point to remark is that all the reducible contributions to the correlations, i.e. not depending on all the coordinates $\boldsymbol{r}_{1}, \ldots \boldsymbol{r}_{2 n}$, are annihilated by the combination of the structure functions. Inserting (13) into (3) we obtain

$$
\begin{aligned}
S_{2 n} & \simeq \frac{\left(\prod_{i=1}^{2 n} \Delta_{\boldsymbol{r}_{i}, \mathbf{0}}\right) f_{2 n, 0}(\underline{\boldsymbol{r}})}{t^{p_{2 n}}} \int g_{2 n, 0}(\underline{\boldsymbol{\rho}}, 1) C_{2 n}\left(t^{\frac{1}{\gamma}} \underline{\boldsymbol{\rho}}, 0\right) d \underline{\boldsymbol{\rho}} \\
& \propto\left(\frac{r}{t^{1 / \gamma}}\right)^{\zeta_{2 n}}\left\langle\theta^{2 n}\right\rangle(t),
\end{aligned}
$$

where $f_{2 n, 0}$ is the irreducible zero mode in (13) with the lowest dimension. The quantities $\zeta_{2 n}=p_{2 n}$ coincide with the exponents of the structure functions in forced steady states [10]. This justifies the classical notion of quasiequilibrium, i.e. that short-distance scaling laws are the same in forced and decaying situations provided the basic parameters are made appropriately time-dependent.

The analytical solution found for the Kraichnan model permits to capture the basic features of the scalar decay. It is in particular quite clear that the key ingredient for the asymptotic self-similarity of the scalar pdf is the validity of the rescaling property (8). We also performed 


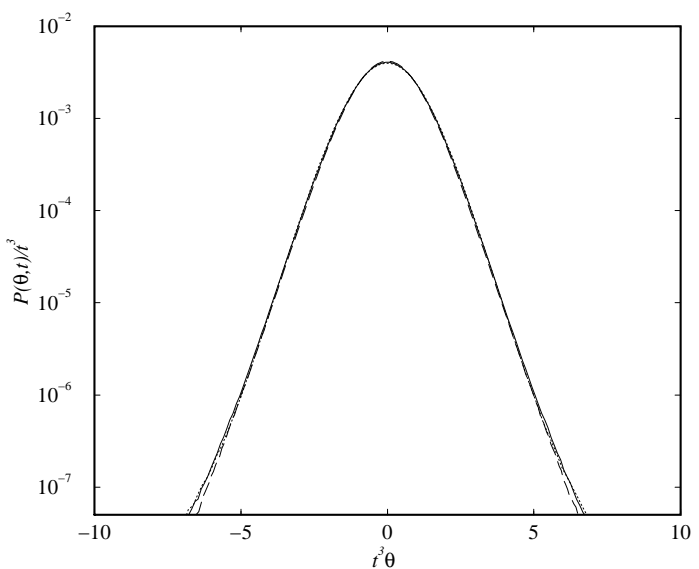

FIG. 3. The rescaled scalar pdf's $v s t^{3} \theta$ for the inverse energy cascade flow at the three times marked by a star in Fig. 2 . The values on the abscissa are normalized by their r.m.s. values.

simulations using a more realistic "inverse energy cascade" 2 -D velocity field which show that such property is not limited to short-correlated velocities and is expected to hold (at least for large enough times) for any self-similar velocity field. Indeed the "inverse cascade" flow is scale-invariant (no intermittency) of exponent $1 / 3$, isotropic and, most important, has finite realistic correlation times [15 17]. The scalar initial distribution is taken Gaussian with correlations decaying exponentially and a correlation length comparable to the velocity injection scale (see Ref. [17] for more details). In Fig. 2 we present the curve of the energy decay showing that the exponent coincides with the value $d / \gamma$ obtained from the Kraichnan model ( $\gamma=2 / 3$ for the inverse cascade flow). The rescaled scalar pdf's $\mathcal{P}(\theta, t) / t^{3}$ for three times in the asymptotic power-law region are presented in Fig. 3 . It is quite clear that the asymptotic distribution is again self-similar and its tails are exponential. The tails are indeed dominated by the rare events governed by Poisson statistics where the scalar is weakly mixed by diffusive effects 18].

For passive scalar decay in an intermittent velocity field, such as a 3-D turbulent flow, the presence of various scaling exponents makes it unlikely that the propagator possesses a rescaling property like (8). The self-similarity of the scalar decay found here might then be destroyed, with the kurtosis and higher-order scalar moments possibly growing indefinitely with time. An even more challenging problem is that of the decay of 3-D turbulent flow which has been frequently addressed, but usually in a phenomenological way and with the emphasis on the law of energy decay (see Refs. 19,20 for a review). Is there self-similar and universal decay as for the problem considered in this paper or is the kurtosis of the velocity growing indefinitely, possibly leading to a highly inter- mittent spatial distribution of the kinetic energy? It is of great interest to address such issues experimentally and by advanced direct numerical simulations.

We are grateful to J. Bec, A. Celani, M. Chertkov, G. Falkovich, K. Gawȩdzki, B. Shraiman and K.R. Sreenivasan for useful discussions. The support by the European Union under Contract HPRN-CT-200000162 and by the National Science Foundation under Grant No. PHY94-07194 is acknowledged. Simulations were performed at IDRIS (no 991226).

[1] B.I. Shraiman \& E.D. Siggia, Nature, 405, 639, (2000).

[2] S. Kida, J. Fluid Mech., 93, 337, (1979).

[3] S.N. Gurbatov, S.I. Simdyankin, E. Aurell, U. Frisch \& G. Toth, J. Fluid Mech., 334, 339, (1997).

[4] L. Frachebourg \& Ph.A. Martin, J. Fluid Mech., 417, 323, (2000).

[5] R.H. Kraichnan, Phys. Rev. Lett., 52, 1016, (1994).

[6] D.T. Son, Phys. Rev. E, 59, 3811, (1999).

[7] E. Balkovsky \& A. Fouxon, Phys. Rev. E., 60, 4164, (1999).

[8] R.H. Kraichnan, Phys. Fluids, 10, 1417, (1967).

[9] S. Corrsin, J. Aeronaut. Sci., 18, 417, (1951).

[10] D. Bernard, K. Gawȩdzki \& A. Kupiainen, J. Stat. Phys., 90, 519, (1998).

[11] U. Frisch, A. Mazzino \& M. Vergassola, Phys. Rev. Lett., 80, 5532, (1998).

[12] U. Frisch, A. Mazzino, A. Noullez \& M. Vergassola, Phys. Fluids, 11, 2178, (1999).

[13] G. Eyink \& J. Xin, J. Stat. Phys., 100, 679, (2000).

[14] V. Hakulinen, "Passive Advection and the Degenerate Elliptic Operators $M_{n}$ ", preprint, (2000).

[15] L. Smith \& V. Yakhot, Phys. Rev. Lett., 71, 352, (1993).

[16] J. Paret \& P. Tabeling, Phys. Fluids, 10, 3126, (1998).

[17] G. Boffetta, A. Celani \& M. Vergassola, Phys. Rev. E, 61, R29, (2000).

[18] B.I. Shraiman \& E.D. Siggia, Phys. Rev. E, 49, 2912, (1994).

[19] A.S. Monin \& A.M. Yaglom, Statistical Fluid Mechanics, Vol. 2, ed. by J. Lumley, MIT Press, Cambridge, Mass., (1975).

[20] U. Frisch, Turbulence. The Legacy of A.N. Kolmogorov, Cambridge Univ. Press, Cambridge, (1995). 